\title{
Study on Automatic Identification Technology of Greenhouse Tomato Pests and Diseases based on Machine Learning
}

\author{
Zhiheng Song ${ }^{1, a}$, Hang Zhang ${ }^{1, b}$, Naixiang $\mathrm{Li}^{1, \mathrm{c}}$ \\ ${ }^{1}$ College of Computer and Information Engineering, Tianjin Agricultural University, Tianjin, 300384 \\ a zhihengsong@163.com, ${ }^{\mathrm{b}}$ zhanghang@tjau.edu.cn, ${ }^{\mathrm{c}}$ linx@126.com
}

Keywords: Automatic Identification, Greenhouse, Tomato

\begin{abstract}
Tomato cultivation in solar greenhouse is a relatively common pattern of protected land planting, with good economic benefits, but the environmental conditions of the greenhouse are conducive to the growth of pathogens, the tomato disease is aggravated, and the greenhouse is relatively closed. Pests such as peach aphid are commonly occurred. The main pests and diseases of greenhouse tomatoes were preliminarily summarized, and corresponding prevention and control measures were proposed for various pests and diseases.
\end{abstract}

\section{Introduction}

With the improvement of people's living standards, people's demand for the quality and variety of vegetables, fruits and flowers is getting higher and higher, especially the anti-season products are favored. Because greenhouses can provide a good environment for growing vegetables and fruits, greenhouse greenhouse production is becoming more and more common, and accurate identification and timely treatment of vegetables, fruits, pests and diseases in greenhouses is particularly important. Artificial methods to identify plant pests and diseases, mainly using color to describe the disease symptoms of plants, there is no quantitative standard. Affected by human factors, and some are not obvious, uncommon diseases are difficult to identify. Therefore, an automatic pest identification system that can be applied to production practice is particularly important.

The greenhouse is one of the core technologies of facility agriculture. The disease identification of crops in greenhouses is mainly observed and sprayed by workers. In China, up to 250,000 tons of chemical pesticides are used every year to control agricultural pests and diseases. The large-scale use of pesticides enhances the resistance of pests and diseases, resulting in crop yield reduction and severe damage to ecological balance. Crop diseases are mostly reflected on leaves or stems. Botanists or greenhouse growers often use empirical knowledge to judge the type and extent of crop damage, and the complexity of visual patterns and the subjectivity of human judgment can cause misjudgments. In order to minimize the spraying of pesticides and accurately identify the disease types on the leaves and stems of the crops, it is necessary to accurately and quantitatively study the symptoms of pests and diseases that can be observed by the naked eye.

\section{Method flow}

Taking tomato leaf disease in greenhouses as the main research object, computer image processing technology was used to image pretreatment, feature extraction and disease identification of diseased tomato leaves. Visual $\mathrm{C}++6.0$ was used as a development tool under Windows XP platform to prepare image pre-preparation. Processing, segmentation, feature extraction, and disease determination procedures ultimately result in a complete image processing system. Image sample: The picture of tomato leaf disease (40 pieces of each of the three diseases) used in this study was from the Internet. The main process: using the difference in color and shape characteristics of the diseased parts of tomato leaves, the disease of tomato is determined by statistical methods. In the course of the research. 
Since the picture size, specifications, background, etc. are not completely unified, the picture should be cut to a certain extent to reduce the interference of external conditions such as background and light. In this study, two types of characteristics (color features and shape features) of tomato leaf diseases were extracted, so two kinds of image preprocessing methods were needed: for the extraction of color features, there are various pretreatment methods, and the large veins of the leaves themselves are not conducive to The color feature of the diseased part is extracted, so the blade image should be filtered and denoised. The linear filtering method has low-pass characteristics, which removes noise and blurs the edges of the image, which destroys the original features of the image. The median filtering method can remove noise and protect the edge of the image. It is a nonlinearity. Processing method. When extracting shape features, in order to reduce noise and protect the edge information of the lesion for subsequent image segmentation, a suitable preprocessing method should be selected. This study compared a variety of pre-processing methods: image linear filtering, smoothing, Laplacian and other methods will destroy the contour of the image lesions, not suitable for this study; median filtering is a nonlinear smoothing Technique, which sets the gray value of each pixel to the median value of the gray values of all pixels in a neighborhood window at that point, so that the surrounding pixel values are close to the true value, and the isolated noise points are eliminated while protecting The edge of the image; the image enhancement method (via a high-pass filter) can also highlight the outline of the image, allowing the shape and perimeter of the contour to be measured. Through experimental comparison, both methods can effectively realize the image edge information needed for shape feature extraction and effectively remove noise. However, in order to optimize the software and reduce the amount of calculation, this study selects the median filtering that is consistent with the color feature preprocessing.

Image segmentation is an important process in image processing and one of the most basic techniques in image processing. The quality of image segmentation determines the ultimate success of image processing. At present, the commonly used image segmentation techniques include: otsu threshold segmentation method, edge detection based segmentation algorithm, neural network based segmentation algorithm and morphology based segmentation algorithm. Among them, the otsu threshold segmentation method is simple to calculate and is not affected by image contrast and brightness under certain conditions, and is considered as the optimal method for automatic threshold selection. After many experimental comparisons, the otsu threshold segmentation method can effectively separate the lesion area from the non-lesion area, and the effect is ideal. The otsu threshold segmentation method is also called the Dajin method, which is the maximum inter-class variance method. It divides the image into two parts, the background and the target, according to the grayscale characteristics of the image. The greater the variance between the lesions and the non-lesional regions, the greater the difference between the two parts of the image; the misclassification between the lesion and the non-lesional area will result in a smaller inter-class variance between the two parts. Therefore, the segmentation that maximizes the variance between classes means that the probability of misclassification is minimal.

\section{Feature extraction}

After the threshold is determined, the lesion is separated from the leaf. Since the edge contour information of the lesion is drawn, by reloading the image, the intersection information of the lesion area and the normal leaf is retained, thereby separating the lesion site. The color and shape characteristics of the lesion were extracted, and the number of lesions was counted. The extracted shape features are: perimeter, area, and circularity. Figure 2 Image of the image after segmentation of the median filtered image of the early pathogen image. Figure 3 Image of the median filtered image of the leaf mold pathogen Luv coordinates Image after image segmentation Fig. 4 Image complexity, elongation, eccentricity and sphericity after image segmentation in the Luv coordinate of the median filtered image of the late blight map. For color images, there are many common color spaces: R G B, HIS, Ohta, Luv, etc. Luv color space full name CIE 1976 (L*, u*, v*) (also known as CIELUV) color space, $\mathrm{L}^{*}$ for object brightness, $\mathrm{u}^{*}$ and $\mathrm{v}^{*}$ for color. The naked eye has a 
relatively complex nonlinear relationship between the visual color of the color and the physical value of the color stimuli, and the color image processing needs to have a better linear chromaticity coordinate with the human vision. Luv coordinates have a good linear relationship with vision, and can be used for color image processing to get better results.

\section{Pest identification and prevention}

Worried. The greenhouse whitefly is a homoptera family. Greenhouse whitefly is one of the most common pests in cultivated cultivation, and it can kill almost all vegetables. Adults and nymphs suck on plant juice, and the leaves of the victim are chlorotic, yellow, wilting, and even the whole plant is dead. Adult body length $1 \sim 1.5 \mathrm{~mm}$, light yellow, wing covered with white wax, commonly known as "small white moth". Under greenhouse conditions, more than 10 generations can occur in a year, and they live in the leaves and spawning of young leaves. The population of whitefly mites continues to develop from spring to autumn, reaching a peak in autumn. In the northern region, due to the close connection and alternating of greenhouse and open vegetable production, the whitefly annihilation can occur.

Prevention and treatment. The prevention and control of whitefly mites should be based on agricultural control, strengthen cultivation management, cultivate non-worm seedlings as the main measure, rationally use chemical pesticides, and actively carry out biological control and physical control. It is recommended that the non-solanthines that are not suitable for eating greenhouses in the greenhouse are less resistant to low-temperature vegetables and reduce the cultivation area of tomatoes. This is not only bad for the occurrence of whitefly, but also greatly saves energy. Before the seedlings, completely kill the residual whitefly, clean up the weeds and residual plants, and add nylon yarns in the vents to control the foreign insect source and cultivate the insect-free seedlings. Avoid mixing cucumber, tomato and kidney beans, so as to avoid creating a good living environment for whitefly, and increase the damage. You can also use 10\% oxaloin 1000 times for drug control, and control once every 7 days for 2 times.

Worried. Helicoverpa armigera belongs to the family Lepidoptera. Helicoverpa armigera is the main pest of solanaceous vegetables. The larvae eat buds, flowers and fruits, and also damage the stems, leaves and buds. When the flower bud is damaged, the leaves are opened and turn yellow-green, and fall off after 2 to 3 days. Young fruit is often eaten or caused to rot and fall off. Although mature fruit is only eaten part of the flesh, because the pupil is in the pedicle, it is easy to cause rain and bacteria to invade and cause decay. Therefore, a large number of fruits will cause the fruit to rot and fall off. resulting in a reduction in production. Helicoverpa armigera has a body length of 14 to $18 \mathrm{~mm}$ and a wingspan of 30 to $38 \mathrm{~mm}$, which is taupe. Helicoverpa armigera overwinters in the soil. In late May, it is the peak of spawning, and the first generation of larvae is the damage period from late May to late June, and the first generation from the end of June to the beginning of July. Helicoverpa armigera is a thermophilic pest. When the temperature in early summer is stable at $20^{\circ} \mathrm{C}$ and $5 \mathrm{~cm}$, the ground temperature is stable above $23^{\circ} \mathrm{C}$, and the wintering cockroach begins to feather. Adults lay eggs at a temperature above $23{ }^{\circ} \mathrm{C}$, and rarely spawn at $20^{\circ} \mathrm{C}$. Larval development is most suitable at 25 to $28^{\circ} \mathrm{C}$ and relative humidity of $75 \%$ to $90 \%$. In the north, the influence of humidity on larval development is more significant. When the monthly rainfall is above $100 \mathrm{~mm}$ and the relative humidity is above $70 \%$, it is more harmful. Prevention and treatment. The land should be ploughed before winter to reduce the source of overwintering insects. According to the insect condition report, in the period of spawning of cotton bollworm, combined with pruning, the eggs are removed. 3 to 4 days after the peak of adult spawning, when the number of eggs reaches 20 to 30, the drug should be started. If there are more than 5 larvae, the drug should be continued. Generally, when the first spike of the tomato grows to the size of the egg, the medicine is started once a week, and the prevention and treatment is continued for 3 to 4 times. Available 2. 5\% cyhalothrin 5000 times solution, 2. 5\% deltamethrin 2000 times solution, sprayed once every 6 days. 


\section{Acknowledgements}

Fund Project: Tianjin Agricultural Science and Technology Achievements Transformation and Extension Project, Integrated Application of Forest Information Disease Early Warning, Diagnosis and Prevention and Control Core Information Technology, Project No. 201704070

\section{References}

[1] Liu Yuanbo, Xue Li, Xian Zhengyi. Discussion on Program Design of Programmable Controller PLC Control System[J]. Gold.2011,32(4):34-36.

[2] Lan Li, Li Hong. Integration of Profinet and Fieldbus [J]. Fieldbus Technology Application 200, 2007, 23(8): 33-34.

[3] Zhang Ning, Liu Wenping. Review of plant leaf recognition technology based on image analysis [J]. Computer Application Research, 2011, 28(11): 4001-4007.

[4] Liu Huanjun, Wang Yaonan, Duan Feng. Image acquisition technology in machine vision [J]. Computer and Information Technology, 2003, 1(1): 18-21.

[5] Yang Ming, Song Lihua. Application of improved fast median filtering algorithm in image denoising [J]. Surveying and Mapping Engineering, 2011, 20 (3): 65-69 